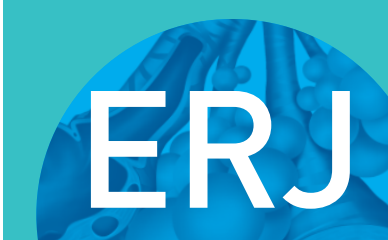

open research

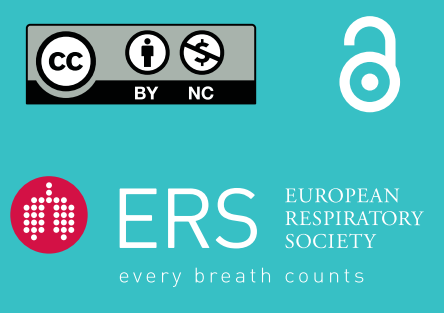

\section{Oral corticosteroid-sparing effects of reslizumab in the treatment of eosinophilic granulomatosis with polyangiitis}

\author{
To the Editor:
}

Eosinophilic granulomatosis with polyangiitis (EGPA) is a rare but devastating vasculitis characterised by perivascular eosinophilic inflammation, severe asthma, peripheral eosinophilia and sinonasal disease, frequently complicated by cardiac, neurological or renal involvement $[1,2]$. The mainstay of therapy for EGPA is use of systemic corticosteroids (OCS), with or without concomitant immunosuppression with methotrexate, azathioprine, cyclophosphamide or rituximab $[3,4]$. The long-term use of these agents is associated with significant drug-related morbidity and the risk of relapse in EGPA patients remains significant despite treatment [1, 5]. Interleukin (IL)-5 is a critical cytokine regulating eosinophil development, migration and activation [2]. In EGPA, high doses of the IL-5 neutralising antibody mepolizumab lead to improved disease control and reduced requirement for OCS therapy, with an excellent safety profile $[6,7]$. Reslizumab is another IL-5 neutralising antibody currently licensed for the treatment of severe eosinophilic asthma [8]; however, there are - to our knowledge - no published data exploring the utility of reslizumab in the management of EGPA. Here, we report clinical and patient-reported outcomes in a cohort of treatment-refractory, OCS-dependent EGPA patients with severe asthma commenced on reslizumab.

Patients with established EGPA and severe eosinophilic asthma requiring long-term continuous OCS to maintain disease control, in whom prior OCS dose weaning efforts had failed, were commenced on 4-weekly reslizumab infusions at a dose of $3 \mathrm{mg} \cdot \mathrm{kg}^{-1}$. All patients were attending a dedicated EGPA clinic run jointly between pulmonary and rheumatology physicians at our tertiary care centre. OCS doses were reviewed during each 4-weekly consultation and adjusted if deemed appropriate by the treating physician. Clinical data including OCS dose, lung function, exhaled nitric oxide fraction $\left(F_{\mathrm{ENO}}\right)$ and need for hospitalisation were recorded at baseline and following 48 weeks of treatment, as were the patient-reported outcome measures, the seven-item Asthma Control Questionnaire (ACQ7) and the mini-Asthma Quality of Life Questionnaire (mini-AQLQ).

Nine patients with OCS-dependent EGPA and severe eosinophilic asthma were commenced on reslizumab between April 2017 and February 2018. They were predominantly middle-aged (mean \pm SD $48.2 \pm 11.7$ years), $44 \%$ were female and all had adult-onset asthma. In keeping with previously described EGPA cohorts, $22 \%$ were anti-neutrophil cytoplasmic antibody positive and four (44\%) had histological confirmation of EGPA. All patients had paranasal sinus disease, $44 \%$ had neurological symptoms of EGPA and 33\% had cardiac involvement. Patients were receiving moderate- to high-dose OCS (mean daily prednisolone dose $23.4 \pm 9.1 \mathrm{mg} \cdot \mathrm{day}^{-1}$, all patients were on $\geqslant 10 \mathrm{mg} \cdot \mathrm{day}^{-1}$ ). All patients had received treatment with at least one other immunomodulatory agent (methotrexate, 44\%; rituximab, 22\%; mycophenolate, 22\%; azathioprine/mepolizumab/intravenous immunoglobulin/tacrolimus, 11\% each). Despite ongoing OCS

$@$ ERSpublications

Blockade of interleukin-5 with reslizumab appears to have significant oral corticosteroid sparing effects in patients with eosinophilic granulomatosis with polyangiitis and severe eosinophilic asthma http://bit.ly/2D2yYSK

Cite this article as: Kent BD, d'Ancona G, Fernandes M, et al. Oral corticosteroid-sparing effects of reslizumab in the treatment of eosinophilic granulomatosis with polyangiitis. ERJ Open Res 2020; 6: 00311-2019 [https://doi.org/10.1183/23120541.00311-2019].

Copyright $\odot$ ERS 2020. This article is open access and distributed under the terms of the Creative Commons Attribution NonCommercial Licence 4.0. 
therapy, symptom scores were suggestive of suboptimally controlled disease (ACQ7, 2.1 \pm 2.1 ; mini-AQLQ, 4.6 \pm 1.8$)$. $F_{\mathrm{ENO}}$ was suggestive of persistent airway inflammation $(61.4 \pm 53.3 \mathrm{ppb})$.

Following 48 weeks of treatment, there was a significant reduction in mean maintenance OCS dose, from a prednisolone equivalent of $23.4 \pm 9.1 \mathrm{mg} \cdot \mathrm{day}^{-1}$ to $5.9 \pm 4.8 \mathrm{mg} \cdot \mathrm{day}^{-1}(\mathrm{p}<0.001)$ (figure 1a). All patients had $\mathrm{a} \geqslant 50 \%$ maintenance OCS dose reduction and seven $(78 \%)$ had their doses reduced to or below the $7.5 \mathrm{mg} \cdot$ day $^{-1}$ prednisolone threshold suggested by the European League Against Rheumatism as being consistent with remission [9], whilst two patients were able to completely stop OCS. This occurred alongside an improvement in mini-AQLQ score from $4.6 \pm 1.8$ to $5.9 \pm 1.6(\mathrm{p}=0.001)$ (figure $1 \mathrm{~b})$ and a non-statistically significant improvement in ACQ7 score from $2.1 \pm 2.1$ to $1.5 \pm 1.8$ ( $p=0.081$ ) (figure 1c). No significant changes were observed in absolute forced expiratory volume in $1 \mathrm{~s}\left(\mathrm{FEV}_{1}\right)\left(\Delta 0.11 \pm 0.43 \mathrm{~L} \cdot \mathrm{s}^{-1}\right.$, $\mathrm{p}=0.465), \mathrm{FEV}_{1} \%$ predicted $(\Delta 2.4 \pm 15.2 \%, \mathrm{p}=0.654)$ or Birmingham Vascular Activity Score $(6.7 \pm 4.1$ at week $0,4.1 \pm 2.0$ at week 48; $\mathrm{p}=0.054)$. Despite the reduction in OCS exposure, peripheral eosinophil counts remained suppressed $\left(\Delta-0.05 \pm 0.12 \times 10^{9}\right.$ per $\left.\mathrm{L}, \mathrm{p}=0.275\right)$, no significant change in $F_{\mathrm{ENO}}$ occurred $(\Delta 6.7 \pm 60.0 \mathrm{ppb} ; \mathrm{p}=0.748)$, and no increases in exacerbation frequency or hospitalisation were seen. No treatment-limiting adverse effects were seen, although one patient reported myalgia following reslizumab infusion and one had an apparent progression of peripheral neuropathic symptoms despite otherwise adequate disease control.

Effective and safe new treatments for EGPA are needed to supplement a therapeutic armamentarium that is of variable efficacy, and that is associated with significant treatment-related toxicity, opportunistic infections and malignancy $[3,10]$. Modulating eosinophilic inflammation via blockade of IL-5 in patients with EGPA is an intuitively attractive strategy: it combines mechanistic plausibility with apparent clinical effectiveness, and an excellent medium-term safety profile in EGPA and severe asthma [7, 8].

Our data suggest that reslizumab may be a therapeutic option in EGPA patients with concomitant severe eosinophilic asthma, facilitating what appears to be a significant reduction in OCS exposure in patients with refractory, suboptimally controlled disease. The reduction in OCS dose in these patients would seem potentially clinically, as well as statistically, significant. Reslizumab is not a panacea, however: no significant improvement in overall BVAS scores was seen, suggesting that reslizumab may be less effective in controlling extrapulmonary manifestations of EGPA, such as neuropathy, than in achieving control of airway disease. This seems consistent with the recently published randomised controlled trial (RCT) of high-dose mepolizumab, wherein a proportion of patients did not achieve disease remission despite a significant improvement in the overall population [7].

This report has some important limitations. Although these data were collected prospectively, this was not a clinical trial and is consequently open to bias. The absence of a control group means that we cannot discount the possibility of a placebo effect or of regression to the mean causing reductions in OCS dose and improvements in quality of life scores. However, our observations are consistent with prior reports of other methods of IL-5 blockade in EGPA, and in an unwell patient cohort, the changes in OCS dose were significantly in excess of those in the placebo arm of the recent mepolizumab RCT [7]. Finally, our patients had a persistent severe asthma EGPA phenotype, and reslizumab may be less effective in modulating extrapulmonary, vasculitic disease phenotypes.
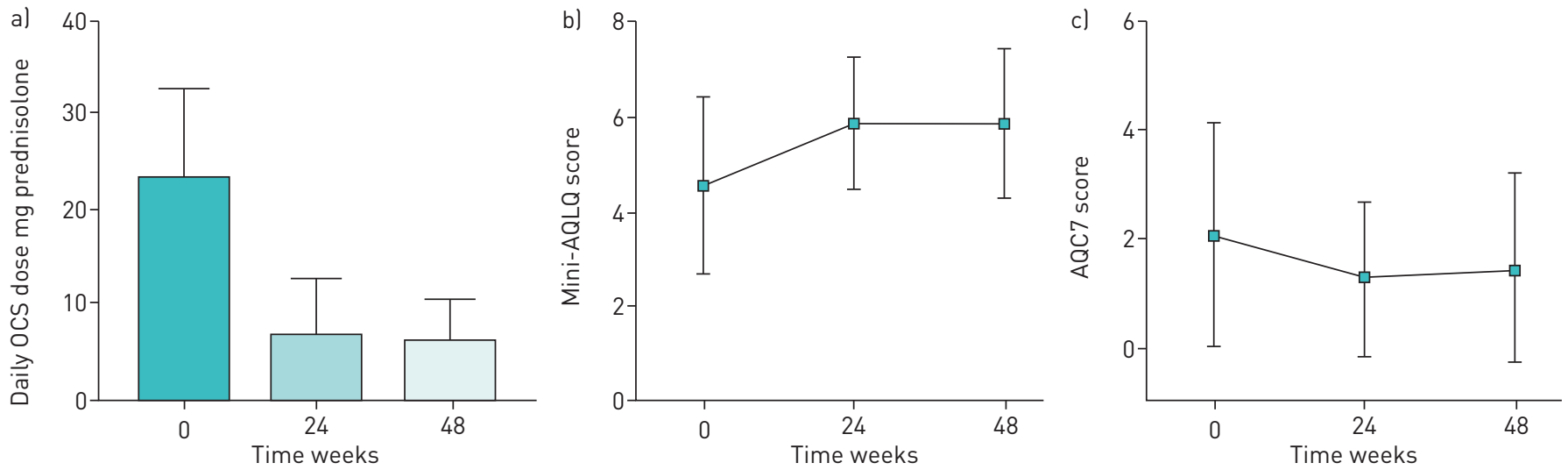

FIGURE 1 Changes in a) oral corticosteroid (OCS) dose ( $p<0.001)$, b) mini-Asthma Quality of Life Questionnaire (mini-AQLQ) $s c 0 r e(p=0.001)$ and c) seven-item Asthma Control Questionnaire (ACQ7) score ( $p=0.081)$ following 48 weeks of treatment with reslizumab. Data are presented as mean \pm SD. 
In summary, in a cohort of EGPA patients with severe eosinophilic asthma requiring continuous OCS to maintain disease control, 48 weeks of treatment with intravenous reslizumab was associated with a significant reduction in OCS use, alongside improvements in patient reported outcomes.

Brian D. Kent ${ }^{1,2}$, Grainne d'Ancona ${ }^{1,2}$, Mariana Fernandes ${ }^{1}$, Linda Green ${ }^{1}$, Cris Roxas ${ }^{1}$, Louise Thomson ${ }^{1}$, Alexandra M. Nanzer ${ }^{1,2}$, Joanne Kavanagh ${ }^{1}$, Sangita Agarwal $^{3}$ and David J. Jackson $\oplus^{1,2}$

${ }^{1}$ Guy's Severe Asthma Centre, Guy's and St Thomas' Hospitals, London, UK. ${ }^{2}$ Asthma UK Centre, King's College London, London, UK. ${ }^{3}$ Dept of Rheumatology, Guy's and St Thomas' Hospitals, London, UK.

Correspondence: Brian Kent, Dept of Respiratory Medicine, St James's Hospital, Dublin 8, Ireland. E-mail: Briankent@physicians.ie

Received: 13 Nov 2019 | Accepted: 18 Nov 2019

Author contributions: B.D. Kent, G. d'Ancona, S. Agarwal and D.J. Jackson conceived and designed the analyses. B.D. Kent, M. Fernandes, L. Green, C. Roxas and J. Kavanagh performed data acquisition. All authors contributed to the writing and critical review of the manuscript.

Conflict of interest: B.D. Kent reports an educational travel grant from Teva Pharmaceuticals during the conduct of the study. G. d'Ancona reports an educational travel grant and speaker fees from Teva Pharmaceuticals during the conduct of the study. M. Fernandes has nothing to disclose. L. Green has nothing to disclose. C. Roxas has nothing to disclose. L. Thomson has nothing to disclose. A.M. Nanzer reports speaker fees from Teva Pharmaceuticals during the conduct of the study. J. Kavanagh has nothing to disclose. S. Agarwal reports grants and personal fees from Teva Pharmaceuticals, during the conduct of the study. D.J. Jackson reports and educational travel grant and speaker fees from Teva Pharmaceuticals during the conduct of the study.

\section{References}

1 Comarmond C, Pagnoux C, Khellaf M, et al. Eosinophilic granulomatosis with polyangiitis (Churg-Strauss): clinical characteristics and long-term followup of the 383 patients enrolled in the French Vasculitis Study Group cohort. Arthritis Rheum 2013; 65: 270-281.

2 Khoury P, Grayson PC, Klion AD. Eosinophils in vasculitis: characteristics and roles in pathogenesis. Nat Rev Rheumatol 2014; 10: 474-483.

3 Groh M, Pagnoux C, Baldini C, et al. Eosinophilic granulomatosis with polyangiitis (Churg-Strauss) (EGPA) Consensus Task Force recommendations for evaluation and management. Eur J Intern Med 2015; 26: 545-553.

4 Ribi C, Cohen P, Pagnoux C, et al. Treatment of Churg-Strauss syndrome without poor-prognosis factors: a multicenter, prospective, randomized, open-label study of seventy-two patients. Arthritis Rheum 2008; 58: 586-594.

5 Cottin V, Bel E, Bottero P, et al. Respiratory manifestations of eosinophilic granulomatosis with polyangiitis (Churg-Strauss). Eur Respir J 2016; 48: 1429-1441.

6 Moosig F, Gross WL, Herrmann K, et al. Targeting interleukin-5 in refractory and relapsing Churg-Strauss syndrome. Ann Intern Med 2011; 155: 341-343.

7 Wechsler ME, Akuthota P, Jayne D, et al. Mepolizumab or placebo for eosinophilic granulomatosis with polyangiitis. N Engl J Med 2017; 376: 1921-1932.

8 Castro M, Zangrilli J, Wechsler ME, et al. Reslizumab for inadequately controlled asthma with elevated blood eosinophil counts: results from two multicentre, parallel, double-blind, randomised, placebo-controlled, phase 3 trials. Lancet Respir Med 2015; 3: 355-366.

9 Hellmich B, Flossmann O, Gross WL, et al. EULAR recommendations for conducting clinical studies and/or clinical trials in systemic vasculitis: focus on anti-neutrophil cytoplasm antibody-associated vasculitis. Ann Rheum Dis 2007; 66: 605-617.

10 Mukhtyar C, Guillevin L, Cid MC, et al. EULAR recommendations for the management of primary small and medium vessel vasculitis. Ann Rheum Dis 2009; 68: 310-317. 